\title{
ALIMENTOS PROCESSADOS E MINIMAMENTE PROCESSADOS PRODUZIDOS POR AGRICULTORES FAMILIARES DA CIDADE DE NERÓPOLIS
}

Processed and minimally processed foods by family farmers from Nerópolis city

Alimientos procesados minimamiente procesados producidos por agricultores familiares de la ciudad de Nerópolis

\section{Daniel Lucino Silva dos Santos ${ }^{1}$, Graciella Corcioli², Yamira Rodrigues de Souza}

Barbosa $^{3}$.

${ }^{1}$ Discente no Curso de Engenharia de Alimentos, Universidade Federal de Goiás -GO, Brasil.

${ }^{2}$ Docente do Curso de Graduação em Agronomia, Universidade Federal de Goiás -GO, Brasil.

${ }^{3}$ Doutoranda no Programa de Pós-Graduação em Ciências Sociais em Desenvolvimento, Agricultura e Sociedade, Universidade Federal Rural do Rio de Janeiro (CPDA/UFRRJ), Brasil.

*Correspondência: Universidade Federal de Goiás- Escola de Agronomia, Avenida Esperança, s/n. Campus Samambaia. CEP: 74.690-900 e-mail daniellucino07@gmail.com

\section{RESUMO}

Com o crescimento do consumo de alimentos processados e a associação destes produtos ao favorecimento de desenvolvimento de doenças crônicas como a hipertensão e obesidade, os produtos minimamente processados aparecem como uma alternativa mais saudável aos consumidores. Este artigo tem por objetivo o estudo sobre os alimentos processados e minimamente processados por produtores familiares da cidade de Nerópolis-GO enfatizando a utilização das boas práticas de fabricação e utilização de máquinas durante o processamento dos produtos. A pesquisa foi realizada em duas etapas: a primeira envolveu a aplicação de questionários à 232 estudantes do ensino médio de escolas públicas do município; na segunda etapa foram realizadas entrevistas com 30 agricultores familiares do município. Os resultados apontam que 4,65\% da população do município moram no meio rural que desenvolvem as seguintes atividades: $60 \%$ trabalham com a criação de animais; $20 \%$ produzem leite e; $20 \%$ desenvolvem atividades agrícolas variadas. O alimento mais processado é o leite, que é transformado em queijos e doces. Entre os minimamente processados estão o milho e o alho. Dentre os colaboradores que participam do processamento dos produtos, $52 \%$ afirmam ter conhecimento sobre boas práticas de fabricação.

Palavras-chave: Alimentos minimamente processados. Boas práticas de fabricação em zonas rurais. Qualidade de alimentos.

\begin{abstract}
With the growth of processed food consumption and the association of these products to the fostering of the development of chronic diseases such as hypertension and obesity, minimally processed foods seem to be a healthier alternative to the consumers. This article aims to study and analyze about what the more processed and minimally processed foods by family producers from Nerópolis city, in the state of Goias, are, which market their products in a municipal scale. Also being studied is the knowledge of good manufacturing practices of the production collaborators and the use of machines during the products' processing. To be able to know about the economic activities, two researches were made, the first, involved the use of a survey with 232 high school students, and the second, involved interviews with 30 farmers of the county. The results pointed to a population of 4,65\% that live in rural areas, of these farmers $60 \%$ worked in animal breeding, $20 \%$ in milk production and $20 \%$ in agriculture. The most processed food is milk, for the production of cheese and sweets, and the minimally processed ones are corn and garlic. Between all the collaborators, 52\% had knowledge of good manufacturing practices.
\end{abstract}


Keywords: Minimally processed foods. Good manufacturing practices. Food quality.

\section{RESUMEN}

Este artículo tiene por objetivo el estudio análisis sobre cuáles los alimientos más procesados minimamiente procesados por productores familiares de la ciudad de Nerópolis, el estado de Goiás, que hacen la comercialización de sus productos a escala municipal. También se estudiaron los conocimientos de buenas práticas de fabricación de los colaboradores de producción y uso de máquinas durante el procesamiento de los productos. Para saber sobre actividades económicas se realizaron dos investigaciones, la involucró la utilización de cuestionarios con 232 estudiantes la Enseñanza Media, y la segunda se realizaron entrevistas con 30 agricultores familiares del municipio. Los resultados apuntan a una población del 4,65\% viviente en zonas rurales, de estos agricultores el 60\% tiene como actividad realizada la cría de animales, el $20 \%$ de la producción de leche y el $20 \%$ de la agricultura. El alimento más procesado es la leche para la producción de quesos y dulces, y entre los mínimamente procesados están el maíz y el ajo. Entre los colaboradores el 52\% tiene conocimientos sobre buenas prácticas de fabricación.

Descriptores: Alimientos minimamiente procesados. Buenas práticas de fabricación. Calidad de alimientos.

\section{INTRODUÇÃO}

A agricultura familiar é responsável pela produção da maior parte dos alimentos consumidos no Brasil. Hoffmann (2014) mostra que a produção familiar se apresenta em $84 \%$ do número total de estabelecimentos rurais do país, sendo responsáveis por $87 \%$ da produção de mandioca, $50 \%$ da produção de feijão, $58 \%$ da produção de leite, $50 \%$ da produção de carne de aves e $41 \%$ da produção de milho. Mesmo com a grande produção de alimentos, a falta de conhecimentos e despreparo sobre técnicas para melhor produção de alimentos é grande, gerando problemas de instabilidade econômica e de produção para os agricultores, podendo causar até mesmo o abandono das atividades (FRANÇA, DEL GROSSI, AZEVEDO, MARQUES, 2009).

Paula (2017) observa que apenas a disponibilidade de crédito não é suficiente, sendo necessários mecanismos que garantam a qualidade dos alimentos produzidos e desenvolvimento de programas de comercialização diferenciados, como o Programa de Aquisição de Alimentos (PAA) e Programa Nacional de Alimentação Escolar (PNAE), que promovam a produção de alimentos saudáveis.

Melão (2012) afirma que a implementação de programas voltados para mercados e agricultores familiares é de grande importância para manutenção dos costumes e hábitos alimentares de cada local, como o consumo de alimentos típicos de cada região. Essa prática é necessária para garantir a diversificação da produção atendendo às exigências sanitárias e demais demandas.

Com a associação de maus hábitos alimentares, onde se tem a ingestão de alimentos com altas taxas de gorduras e açúcar, às doenças como a obesidade e a hipertensão (SILVA, 2012), a demanda por alimentos orgânicos e que não passem por várias etapas de processamento (os denominados alimentos ultra processados) é crescente, mostrando novos hábitos alimentares, onde há busca por alimentos que apresentem estrutura natural e sem ser processados (CARVALHO, 2016).

Uma alternativa de consumo são os alimentos minimamente processados, que oferecem praticidade e mantém as características nutricionais e sensoriais quando chegam à mesa do consumidor (MALVEZZI, 2015). Os produtos minimamente processados são alimentos que passam por colheita, transporte, préseleção, classificação, lavagem, sanitização, descascamento ou corte, pesagem e embalo. Não passando por processos como aquecimento ou congelamento (OETTERER, 2010).

Tendo em vista a procura, demanda e comercialização de produtos processados e 
minimamente processados produzidos por agricultores familiares necessita-se de estudos para análise de garantia de qualidade destes alimentos. Magalhães (2011) afirma que é preciso seguir e aplicar as regulamentações, diretrizes federais, estaduais e municipais de boas práticas de fabricação, (BPF) para se ter uma correta manipulação desde a matéria-prima até produto final.

\section{O município de Nerópolis}

Nerópolis está inserida na Região Metropolitana de Goiânia, estando situada a 20 quilômetros de distância da metrópole. Tal proximidade contribuiu para a grande influência que Nerópolis teve no campo tecnológico e de serviços, além do fluxo migratório de renda e serviços entre as duas cidades.

A Região Metropolitana de Goiânia (RMG) foi criada pela Lei Complementar $n^{\circ} 027$ de dezembro de 1999. Atualmente, por meio da Lei Complementar $\mathrm{n}^{\circ} 078$ de 25 de março de 2010, a RMG passou a ser composta por 21 municípios, incluindo o Polo-Goiânia e a cidade de Nerópolis. É importante salientar que segundo os dados do censo de 2010 do Instituto Brasileiro de Geografia e Estatística (IBGE), os municípios da RMG apresentam uma taxa de crescimento populacional de $3,0 \%$ ao ano, sendo superior à de Goiânia, que apresenta 1,79\% de crescimento. As cidades periféricas da RMG, incluindo Nerópolis, a qual apresenta crescimento de $2,37 \%$ ao ano, são chamadas de "cidades dormitórios", em que as pessoas moram, porém, resolvem seus problemas sociais e passam a maior parte de seu tempo na cidade Polo-Goiânia. Nerópolis, pelos dados do censo de 2010 apresenta 95,5 \% de taxa de urbanização, enquanto Goiânia possui 99,6\%. É um município do estado de Goiás, situado na Mesorregião do Centro Goiano, que se estende por $204,2 \mathrm{~km}^{2}$ e conta com 27,341 habitantes, pelos dados do censo do IBGE de
2017. Sua densidade demográfica é de 118,4 habitantes por $\mathrm{km}^{2}$ no território do município. Faz limites com municípios de Ouro Verde de Goiás, Anápolis, Teresópolis de Goiás, Goianápolis, Goiânia, Santo Antônio de Goiás e Nova Veneza (MOYSES,2010).

Assim sendo analisada a polarização dos municípios da Região Metropolitana de Goiânia, temse como objetivo verificar o conhecimento dos agricultores familiares que comercializam alimentos processados e minimamente processados, sobre boas práticas de fabricação de alimentos. Analisando também a utilização de máquinas para a produção dos alimentos e o tempo em que os agricultores atuam comercializado seus produtos no município de Nerópolis, Goiás, descrevendo assim um perfil destes agricultores, que produzem e comercializam seus alimentos no próprio município.

\section{MATERIAL E MÉTODOS}

A primeira pesquisa realizada em novembro de 2017, na cidade de Nerópolis-GO, envolveu a utilização de questionários com 232 estudantes do ensino médio de duas escolas estaduais da cidade de Nerópolis. A pesquisa foi dividida entre entrevistas com os estudantes que residiam no meio rural, e questionários com os estudantes que residiam na zona urbana, portanto, os dados aparecem de maneira separada.

Com os estudantes que moram em zona urbana questionou-se sobre os seus conhecimentos sobre programas para o desenvolvimento rural; possível mobilidade da família entre a cidade e campo; ponto de vista sobre a modernização atual do campo. Com os estudantes que residem na zona rural foram realizadas entrevistas, onde se questionou sobre o conhecimento sobre programas para o desenvolvimento rural no Brasil; quantidade de trabalhadores pertencentes ao núcleo familiar que possuem Carteira de Trabalho e 
Previdência Social (CTPS) assinada; atividade realizada no meio rural e por fim, visão sobre a modernização do campo.

Na segunda pesquisa, realizada em 5 de julho de 2018, realizou-se entrevistas com 30 agricultores familiares que comercializam produtos processados e minimamente processados em feiras realizadas no próprio município. Na pesquisa questionou-se sobre o conhecimento de programas para o desenvolvimento rural; alimentos processados e minimamente processados que são comercializados pelo entrevistado no município; tempo de atuação em atividades comerciais com a venda de alimentos produzidos na sua propriedade rural; conhecimento e aplicação das boas práticas de fabricação pelos colaboradores de produção durante o armazenamento, processamento, e transporte dos alimentos que são comercializados.

\section{RESULTADOS E DISCUSSÃO}

Com relação ao conhecimento sobre programas para o desenvolvimento rural, entre os estudantes residentes na cidade, aproximadamente $27 \%$ tinham algum conhecimento, enquanto $73 \%$ não. O percentual entre estudantes das zonas rurais que desconheciam programas é de $42 \%$, enquanto $58 \%$ conheciam sobre o tema. Índices extramamente baixos, que podem estar relacionados com a falta de aplicações de programas em áreas rurais e também com as taixas de analfabetismo, juntamente com o nível de escolaridade das populações entrevistadas. Sendo que segundo a Pesquisa Nacional por Amostra de Domicílios Contínua (PNAD) realizada em 2017, as taxas de analfabetismo em Goiás são de 4,9\% em pessoas maiores de $15,18,25$ e 40 anos e de 5,2\% para pessoas com mais de 60 anos.

Quanto a aplicação de programas, tem-se como dado de estudo a utilização do Pronaf, que é o principal programa de apoio ao agricultor familiar. A utilização de tal programa tem pouca relevância quando se compara Nerópolis a outros munícios da região metropolitana. Os dados levantados indicam um baixo número de contratos agrícolas e pecuários, com valor total de 366 mil reais aplicados no ano de 2017. Os valores totais dos contratos sofreram queda de investimento a partir de 2016 e não voltaram a aumentar (BANCO CENTRAL DO BRASIL, 2018).

Entre os estudantes que residem na cidade, com relação à existência de familiares que moram no campo bem como o possível hábito de realizar visitas/passeios em zonas rurais, $77 \%$ tem familiares que moram no campo e $79 \%$ costumam fazer visitas a zonas rurais.

Por fim, para os estudantes que moram em zonas rurais, perguntou-se sobre a atividade predominante realizada pela família. Este questionamento apontou que $60 \%$ das famílias realizam a criação de animais, 20\% desenvolvem atividades agrícolas variadas e $20 \%$ produzem leite.

Entre os agricultores familiares que comercializam seus produtos no município de Nerópolis, $41 \%$ afirmam ter conhecimento à cerca dos programas para o desenvolvimento rural.

Os alimentos mais processados no município são o leite, para produção de doces e queijos, guariroba e açafrão, e os produtos minimamente processados, são: alho, couve e milho. Quanto ao conhecimento básico sobre boas práticas, $69 \%$ dos agricultores familiares dizem ter conhecimento e fazer aplicabilidade das normas básicas, porém $86 \%$ não cumprem todas as normas previstas em lei, sendo destacada a dificuldade de custo em fazer-se cumprir todas as exigências estruturais, de armazenamento e transporte dos alimentos até a chegada nas feiras para comercialização.

Os agricultores familiares que comercializam alimentos processados, $72 \%$ dizem fazer a produção 
utilizando utensílios como toucas e luvas e afirmam ter local reservado para a produção de queijos.

O tempo médio de comercialização de produtos pelos agricultores é de 3 (três) anos, o que indica mercado consumidor consolidado para os produtos produzidos em suas propriedades.

Em pesquisa realizada com 144 agricultores familiares dos dezesseis municípios pertencentes ao Território Vale do Rio Vermelho, Medina, Camargo e Silvestre (2018) verificaram que 139 criavam gado; 24 desenvolviam fruticultura e 9 horticulturas. Concluindo que mesmo com o grande potencial de desenvolvimento rural, os agricultores têm sistemas de produção pouco diversificados, tento grande concentração em torno da pecuária leiteira. Essa pouca diversificação pode ser produto da falta de conhecimentos de manuseios de novas técnicas e falta de investimentos para a obtenção de saberes para se fazer a diversificação e produção de produtos artesanais que garantiriam maior valor agregado.

\section{CONCLUSÃO}

Os resultados mostram agricultores que ainda mantém as tradições desde a fundação do município, com cultura de tradição em condimentos e folhas, o que faz-se necessário é um maior conhecimento destes agricultores sobre programas como o PRONAF e outros que são voltados para a agricultura familiar para se ter um maior investimento no setor e assim melhor disseminação de conhecimento, com o aprimoramento dos agricultores tanto em técnicas para aumentar a sua produção como também em investimentos para se poder cumprir todas a normas de boas práticas durante o processo de produção dos seus alimentos que serão comercializados com um mercado que já tem uma cultura e tradição de preferência por produtos com práticas saudáveis de plantio, armazenamento e transporte. Garantindo segurança para os futuros consumidores.
Todos os autores declararam não haver qualquer potencial conflito de interesses referente a este artigo.

\section{REFERÊNCIAS}

BANCO CENTRAL DO BRASIL (BCB) Departamento de Regulação, Supervisão e Controle das Operações do Crédito Rural e do Proagro (Derop). Sistema de Operações do Crédito Rural e do Proagro (Sicor). Disponível em: $<$ http://www.bcb.gov.br/pt-

br/\#!/n/CREDRURAL >. Acesso em: 08 jul. 2018.

CARVALHO S. A. de; ESTRELA P. da COSTA; SILVA A. R. da. Padrão alimentar de adolescentes de uma escola pública. Multi-Science Journal, v. 1, n. 5, P. 70-74, 2016.

FRANÇA, C. G. de; DEL GROSSI, M. E.; AZEVEDO MARQUES, V. P. M. de. O censo agropecuário 2006 e a agricultura familiar no Brasil. Brasília: MDA, 2009.

HOFFMANN, R. A agricultura familiar produz $70 \%$ dos alimentos consumidos no Brasil? Segurança Alimentar e Nutricional, Campinas, v.21, n.1, p.417421, 2014.

INSTITUTO BRASILEIRO DE GEOGRAFIA E ESTATÍSITCA (IBGE). Estimativas da população residente com data de referência $1^{\circ}$ julho de 2017. Diretoria de Pesquisas, Coordenação de População e Indicadores Sociais. Disponível em: <http://cod.ibge.gov.br/2VS1U〉. Acesso em $25 \mathrm{dez}$. 2017.

INSTITUTO BRASILEIRO DE GEOGRAFIA E ESTATÍSITCA (IBGE). Número de estabelecimentos e Área dos estabelecimentos agropecuários por grupos de área total em Goiássérie histórica, 2016 Disponível em: $<$ https://sidra.ibge.gov.br/pesquisa/censoagropecuario/censo-agropecuario-2006/segundaapuracao\#series-temporais>. Acesso em: 31 mar. 2018.

INSTITUTO BRASILEIRO DE GEOGRAFIA E ESTATÍSTICA (IBGE). Primeiros resultados da Pesquisa Nacional por Amostra de Domicílios Contínua. Disponível em: https://ww2.ibge.gov.br/home/estatistica/pesquisas/pe squisa_resultados.php?indicador $=1 \&$ id_pesquisa $=14$ 9. Acesso em: 10 jul. 2018.

MAGALHÃES M. A, DIAS Geruza, MILAGRES Maria P; OTTOMAR M., SOARES Claúdio F. Implantação das boas práticas de fabricação em uma indústria de laticínios da zona da mata mineira. In: 
Congresso Brasileiro de Qualidade. Disponível em< http://www. terraviva. com. br/IICBQL/p005. pdf>. Acesso em: 08 de jul. 2018.

MALVEZZI B. Z; ESCAVANAQUI, Gabrielle R., TEIXEIRA, G; LIMA, Joana Darc M; BUENO, Vanessa G. C; TROVA, Elaine C. V; TROVA, Rosângela V; GONÇALVES, Maria G. C. Pesquisa mercadológica sobre consumo de alimentos minimamente processados - Mogi Mirim, 2015. Revista Universitas, v.8, n.15, p.137-149, 2015.

MEDINA Gabriel, CAMARGO Ricardo, SILVESTRE Wender. Retrato da agricultura familiar em Goiás: relevância, sistemas de produção e alternativas para sua consolidação. In: Agricultura familiar em Goiás: lições para o assessoramento técnico [Recurso eletrônico] / Gabriel Medina (Org.). - 4. ed. - Goiânia: Editora UFG, 2018.

MELÃO, I. B. Produtos sustentáveis na alimentação escolar: o PNAE no Paraná. Caderno IPARDES. Curitiba, v. 2, n.2, p. 87-105, jul./dez. 2012.

MOYSES, Aristides; BORGES, Elcileni M. e CUNHA, Débora F. O estado de Goiás e a Região Metropolitana de Goiânia no Censo 2010. Boletim do Observatório das Metrópoles/INCT-CNPq. Ano III, no 196, p. 8-9. Disponível em: <www.observatoriodasmetropoles.net>. Acesso em: 27 jan. 2018

NERÓPOLIS (GO). Prefeitura. 2017. Disponível em:<http://www.neropolis.go.gov.br/sobre-omunicipio/historia-de-neropolis/>. Acesso em: jan. 2018, 17:31:30.

OETTERER, M.; REGITANO-d'ARCE, M. A. B.; SPOTO, M. H. F. Fundamentos de Ciências e Tecnologia de Alimentos. Barueri: Manole, 2010. 612 p.

PAULA, M. M. de; OLIVEIRA, A. L. SILVA, J. L. G. Promoção da saúde e produção de alimentos na agricultura familiar. Revista Interação Interdisciplinar, v. 01, n. 01, p.50-67, 2017.

SILVA, J.G.; Teixeira, M. L. O.; Ferreira. Alimentação e saúde: sentidos atribuídos por adolescentes. Esc Anna Nery,16 (1):88- 95, 2012. 